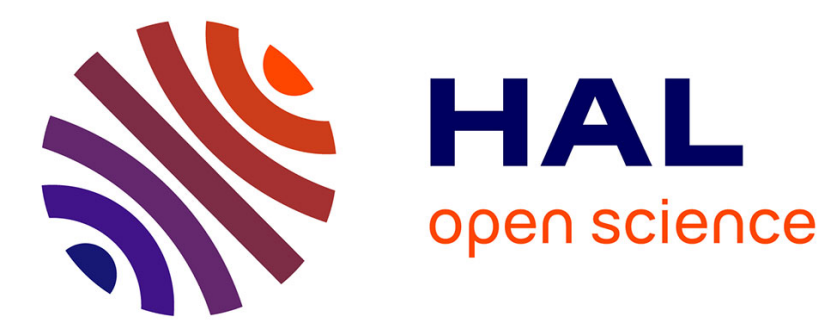

\title{
A Dual-user Teleoperation System with Online Authority Adjustment for Haptic Training
}

Fei Liu, Arnaud Lelevé, Damien Eberard, Tanneguy Redarce

\section{To cite this version:}

Fei Liu, Arnaud Lelevé, Damien Eberard, Tanneguy Redarce. A Dual-user Teleoperation System with Online Authority Adjustment for Haptic Training. 37th IEEE EMBC, IEEE Engineering in Medecine and Biology Society, Aug 2015, Milan, Italy. 10.1109/EMBC.2015.7318574 . hal-01199676

\section{HAL Id: hal-01199676 https://hal.science/hal-01199676}

Submitted on 28 Jan 2016

HAL is a multi-disciplinary open access archive for the deposit and dissemination of scientific research documents, whether they are published or not. The documents may come from teaching and research institutions in France or abroad, or from public or private research centers.
L'archive ouverte pluridisciplinaire HAL, est destinée au dépôt et à la diffusion de documents scientifiques de niveau recherche, publiés ou non, émanant des établissements d'enseignement et de recherche français ou étrangers, des laboratoires publics ou privés. 


\title{
A Dual-user Teleoperation System with Online Authority Adjustment for Haptic Training
}

\author{
Fei Liu, Arnaud Lelevé, Damien Eberard and Tanneguy Redarce
}

\begin{abstract}
This paper introduces a dual-user teleoperation system for hands-on medical training. A shared control based architecture is presented for authority management. In this structure, the combination of control signals is obtained using a dominance factor. Its main improvement is Online Authority Adjustment (OAA) : the authority can be adjusted manually/automatically during the training progress. Experimental results are provided to validate the performances of the system.
\end{abstract}

\section{INTRODUCTION}

Over the last few decades, medical robotic systems have been studied more specifically to perform clinical assistance [1]. Of the various research topics in this field, bilateral teleoperation is intensively tackled. It enables operators to get the feeling of the remote environment by means of force feedback. It has been used in many applications such as Minimally Invasive Surgery (MIS) [2] but also for medical training systems [3].

Dual-user teleoperation is an extension of the traditional bilateral system, which features two users working collaboratively and simultaneously on a shared remote environment. In a hands-on training context, the trainer and the trainee can thus interact remotely with each other. A typical dual-user training system is shown in Fig. 1.

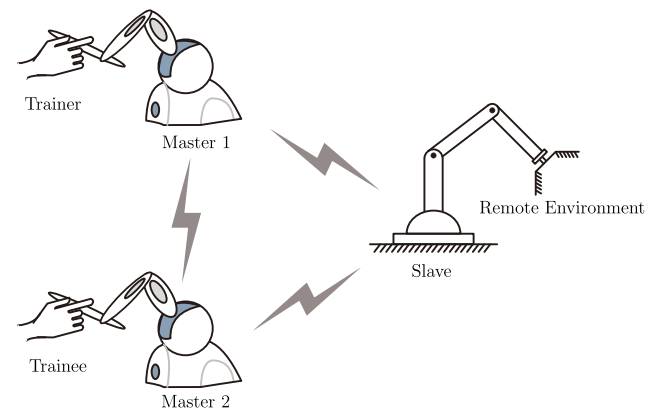

Fig. 1. Scheme of a dual-user teleoperation system

Different architectures have been proposed for dual-user teleoperation systems. The shared control based structure has been mostly developed in [4], [5], [6], [7]. These systems provide haptic feedback to surgeons (masters) through haptic devices such as those depicted in Fig. 1.

The main idea of this architecture is to share the control authority of each user over the slave robot according to a

The authors are with the Laboratory Ampère (CNRS UMR 5005), Université de Lyon, INSA de Lyon, 25 ave J. Capelle Ouest, F69621, Villeurbanne Cedex, France (Emails: \{fei.liu, arnaud.leleve, damien.eberard, tanneguy.redarce\}@insa-lyon.fr).

The authors acknowledge the financial support of the China Scholarship Council (CSC) Scholarship. dominance factor $(\alpha \in[0,1])$. When $\alpha=1$ (resp. 0), the trainer (resp. trainee) has full authority on the trainee's (resp. trainer's) device and the slave. When $0<\alpha<1$, both users share the slave control with a dominance (over the other user) which is function of $\alpha$. The control authority shared between the users is chosen according to their relative level of skills and experience.

For our particular application of surgical training, we needed to design a system enabling leader-follower modes with online authority adjustment behaviors. Suppose a novice is trained from the beginning step by step by a mentor. At first, the trainer sets the system in training mode, which implies that the trainer is the leader while the trainee is the follower. The trainer performs and shows the right trajectories to complete a manual predefined task which involves for the slave to get into contact with the environment. During this demonstration, the trainer is given full force feedback from the slave. Meanwhile, the trainee follows the motion of the trainer on his own haptic device.

During the training progress, the control authority can be shifted towards the trainee $(0<\alpha<1)$ by way of online settings. The trainer can therefore guide the trainee to perform tasks based on shared control authority. Both of them get then a weighted force feedback from the environment.

Once the trainee is sufficiently trained, the control authority is fully assigned to him. In case of emergency (for instance, when the trainee deviates from the right motion, which is risky for the virtual patient), the trainer, by reflex, rectifies the trajectory with its own device which automatically and instantaneously switches back the control authority towards him. The trainee can thus quickly feel the reaction of his mentor and be aware of his own bad movements. The trainer decides later to switch back the authority to the trainee by pressing a dedicated button on the haptic device.

This kind of training system requires online authority adjustment behaviors without disturbing the stability and performance. Only a few papers have considered about this issue despite its importance. In [8], the control authority is adaptively adjusted according to the trainee's expertise. The Virtual Fixtures (VF) incorporated in the system are used as a quantitative measure to evaluate the performance of the trainee. However they require to indicate the maximum desired level of authority of the trainee over the task at first; de facto, the trainer can not change the desired level of authority during the training progress. In [9], the aforementioned shared control authority management is used with a time-varying dominance factor. Still, the proposed method necessitates a bounded first-derivative for the dominance 


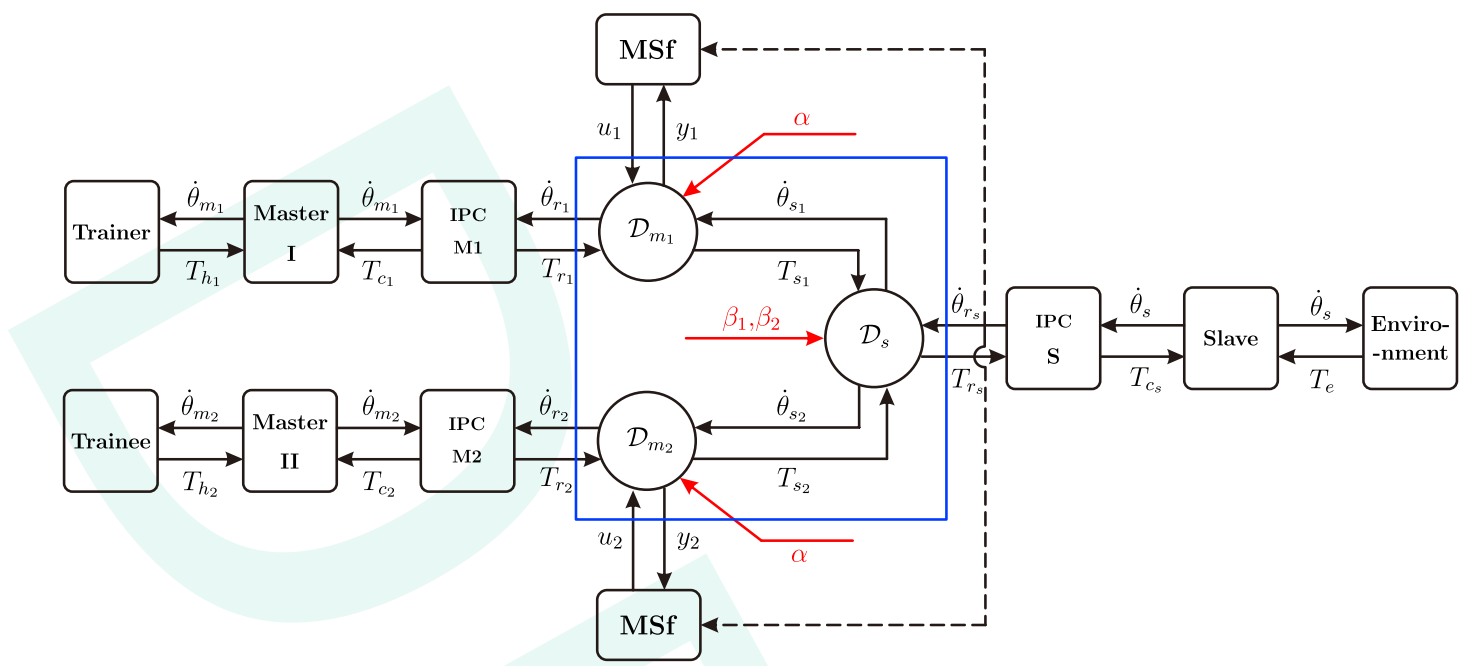

Fig. 2. Global scheme of our dual-user training system. The structure inside the blue solid line is the shared control based architecture.

factor $\alpha(t)$. This involves that it should be designed as a derivable function : this constrains the cases of emergency when the authority needs to be switched suddenly.

In this paper, we study a dual-user haptic training system combined with shared control concept. The Online Authority Adjustment (OAA) is proposed with manual and automatic behaviors. The passivity of the system is preserved using port-Hamiltonian approach. Next section introduces the structure of this system while section II details the OAA mechanism. Section III presents some experiments.

\section{Shared Control Based Dual-User TELEOPERATION}

In this section, we introduce a shared control dual-user training system as shown in Fig. 2.

\section{A. Port-Hamiltonian Approach}

In [10], the port-Hamiltonian approach has been introduced for system dynamic modeling. This approach has been applied both for modeling and controlling bilateral teleoperation system in [11], [12]. It allows to keep track and to control its energy flows. From the point of view of control, one of the advantages of this approach is that the Hamiltonian function, which is usually formed as a Lyapunov function explicitly appears in the dynamics of the system.

The Intrinsically Passive Controller (IPC) formed within Port-Hamiltonian representation, firstly proposed in [13], is passive by itself whatever the values of its parameters. By linking the IPC controller and the master robot through a power-conserving interconnection, one obtains a passive system, whatever the characteristics of the system and the controller parameters. This provides a natural robustness and leaves freedom to tune its parameters for performance and global transparency. In our dual-user system, we use IPC controllers for compliant motion tasks on both masters and the slave.

\section{B. Shared Control Based Architecture}

In order to manage the control authority, three Dirac structures (see [14]) are inserted between the IPC controllers, named $\mathcal{D}_{m_{1}}, \mathcal{D}_{m_{2}}$ and $\mathcal{D}_{s}$. Three dominance factors $\alpha, \beta_{1}, \beta_{2} \in$ $[0,1]$ are introduced to achieve the goal of shared control. As the efforts (forces) and flows (velocities) follow powerconserving interconnection (skew-symmetric representation, see [14]), the behavior of these Dirac structures is passive and lossless. The formulation of $\mathcal{D}_{m_{1}}, \mathcal{D}_{m_{2}}$ and $\mathcal{D}_{s}$ as inputoutput matrices is :

$$
\begin{aligned}
& \mathcal{D}_{m_{1}}:\left(\begin{array}{c}
\dot{\theta}_{r_{1}} \\
T_{s_{1}} \\
y_{1}
\end{array}\right)=\left(\begin{array}{ccc}
0 & \alpha & 1-\alpha \\
-\alpha & 0 & 0 \\
\alpha-1 & 0 & 0
\end{array}\right)\left(\begin{array}{l}
T_{r_{1}} \\
\dot{\theta}_{s_{1}} \\
u_{1}
\end{array}\right) \\
& \mathcal{D}_{m_{2}}:\left(\begin{array}{c}
\dot{\theta}_{r_{2}} \\
T_{s_{2}} \\
y_{2}
\end{array}\right)=\left(\begin{array}{ccc}
0 & 1-\alpha & \alpha \\
\alpha-1 & 0 & 0 \\
-\alpha & 0 & 0
\end{array}\right)\left(\begin{array}{l}
T_{r_{2}} \\
\dot{\theta}_{s_{2}} \\
u_{2}
\end{array}\right) \\
& \mathcal{D}_{s}:\left(\begin{array}{c}
\dot{\theta}_{s_{1}} \\
\dot{\theta}_{s_{2}} \\
T_{r s}
\end{array}\right)=\left(\begin{array}{ccc}
0 & 0 & \beta_{1} \\
0 & 0 & 1-\beta_{2} \\
-\beta_{1} & \beta_{2}-1 & 0
\end{array}\right)\left(\begin{array}{l}
T_{s_{1}} \\
T_{s_{2}} \\
\dot{\theta}_{r s}
\end{array}\right)
\end{aligned}
$$

The relationships between $\alpha$ and $\beta_{1}, \beta_{2}$ are defined as,

$$
\beta_{1}=\left\{\begin{array}{lr}
\alpha, \quad \alpha=1,0 \\
1, & 0<\alpha<1
\end{array} \quad \beta_{2}= \begin{cases}\alpha, & \alpha=1,0 \\
0, & 0<\alpha<1\end{cases}\right.
$$

Thus, we obtain three modes by changing the dominance factor $\alpha$ : training mode $(\alpha=1)$, guidance mode $(0<\alpha<$ $1)$ and evaluation mode $(\alpha=0)$. Notice that the modulated flow source MSf is associated with a velocity signal which is equal to $\dot{\theta}_{r_{s}}$. It is demonstrated (not in this paper for space reason) that these flow sources preserve the passivity of the system as long as both operators and the environment are passive, which is a common assumption in the literature.

\section{Online Authority Adjustment}

In this section, we propose Online Authority Adjustment (OAA) with manual and automatic behaviors. It is an important function during the training progress, for example when emergency situations. 


\section{A. Manual OAA}

The trainer can adjust the dominance factors in real-time, which enables the trainee to get involved in the tasks with a desired level of authority without having to restart the system.

\section{B. Automatic $O A A$}

In evaluation mode $(\alpha=0)$, the trainee imposes the motions on the slave and then the slave on the trainer's haptic device, with a compliant behavior : the trainer can resist to the motion, a small force pushes back the interface towards the slave position. Since the trainee may perform unexpected motions that may require to be rectified instantaneously, when the trainer moves intentionally its device to a different location compared to the slave one, the dominance factor $\alpha$ is instantaneously switched to 1 in order to rectify the slave motion and to make the trainee haptically feel his bad motion.

In order to achieve this automatic behavior, we created a virtual boundary based on the distance between both master positions (see eq. 3). As long as the trainer follows the trainee, the master positions remain inside this boundary; the trainee is able to perform his tasks by himself while the trainer follows the slave motion through his compliant device. As soon as the trainer modifies his device motion, the position error crosses the boundary and $\alpha$ is immediately switched :

$$
d_{\theta_{m}}=\left(\theta_{m_{1}}-\theta_{m_{2}}\right)^{2} \leq B
$$

where $B$ defines the allowed maximum distance between the trainer and the trainee, which indicates the virtual boundary. This value is chosen according to the task to perform, the expected motion precision and the expertise of the trainee. It can be adjusted in real-time.

\section{EXPERIMENTS}

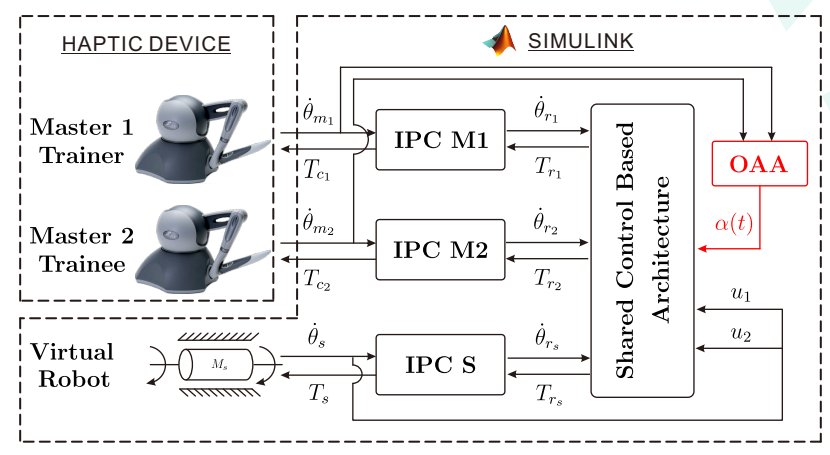

Fig. 3. The experiment setup

To evaluate the performances of this framework, following experiments have been conducted. The haptic training software part was built into MATLAB/Simulink as shown in Fig. 3. Two Sensable ${ }^{\circledR}$ PHANTOM Omni devices (only their first joints) were used as the two masters operated by the trainer and the trainee. The devices' kinematic and dynamic parameters could be found in [15]. A simulated one d.o.f. joint mass robot with friction was set up for the virtual slave. Notice that the slave robot interconnects with a different form of IPC controller proposed in [13], in oder to fit reverse causality interaction with two masters. The explicit equations of the master IPC controller could be found in [13]. The model of the slave IPC controller can be obtained in the same way.

\section{A. Manual $O A A$}

In this experiment, we conducted a manual online authority adjustment. The objective of the trainee during this experiment is to bring the slave device at a target position located at angle $0.3 \mathrm{rad}$. A virtual wall was set at angle $0.5 \mathrm{rad}$. The wall model was :

$$
T_{\text {wall }}= \begin{cases}0, & \theta_{s}<0.5 \mathrm{rad} \\ -k_{w}\left(\theta_{s}-0.5\right), & \theta_{s} \geq 0.5 \mathrm{rad}\end{cases}
$$

where $k_{w}=10 \mathrm{~N} \cdot \mathrm{m} / \mathrm{rad}$ is the wall stiffness and $\theta_{s}$ is the slave robot's joint angle. The hand torques are estimated with a Nicosia observer described in [16]. The tracking of positions, control torques and hand/environment torques are shown in Fig. 4. The experiment time is separated into six phases, i.e. A, B, C, D, E, F, given as Table I. Note the values selected represent a complete training process.

TABLE I

THE EXPERIMENT PHASES

\begin{tabular}{|c|c|c|c|c|c|c|}
\hline Period & $\mathrm{A}$ & $\mathrm{B}$ & $\mathrm{C}$ & $\mathrm{D}$ & $\mathrm{E}$ & $\mathrm{F}$ \\
\hline$\alpha$ & 1 & 0.8 & 0.5 & 0 & 0 & 1 \\
\hline$\beta_{1}$ & 1 & 1 & 1 & 0 & 0 & 1 \\
\hline$\beta_{2}$ & 1 & 0 & 0 & 0 & 0 & 1 \\
\hline Mode & $\mathrm{T}$ & $\mathrm{G}$ & $\mathrm{G}$ & $\mathrm{E}$ & $\mathrm{E}$ & $\mathrm{T}$ \\
\hline Wall Contact & $\mathrm{N}$ & $\mathrm{N}$ & $\mathrm{N}$ & $\mathrm{N}$ & $\mathrm{Y}$ & $\mathrm{N}$ \\
\hline
\end{tabular}

During the training mode (phase A), the trainer acts as the leader (see Fig. 4) while the trainee follows the trainer. In phases $\mathrm{B}$ and $\mathrm{C}$ (guidance mode), both the trainer and the trainee control the slave with respect to their authorities. The slave doesn't reach the target due to the unpracticed motion of the trainee. It is a weighted position of both users. The hand torques are inversely proportional to the authority as well (disregard the torque direction). In the guidance mode, the trainee is involved in the task with a certain level of authority. In phases D and E, the trainee is given full authority. In order to show the unskilled motion, the trainee leads the slave to a wall contact at time $41.2 \mathrm{~s}$ (phase E). During this phase, the trainee feels a full reaction torque generated by the environment, which indicates good transparency. In evaluation mode, the trainer cannot affect at all the slave (by trying to pull the slave in the opposite direction). The slave is in contact with the wall until the authority is manually switched back to the trainer at time 52.8s. As it can be seen in the torque plot, the trainee's applied torque matches the torque the environment applies on the slave during F phase. After then, the slave and the trainee follow the trainer's movements. Consequently, both user experienced different authority levels with manual OAA settings during this experiment. 

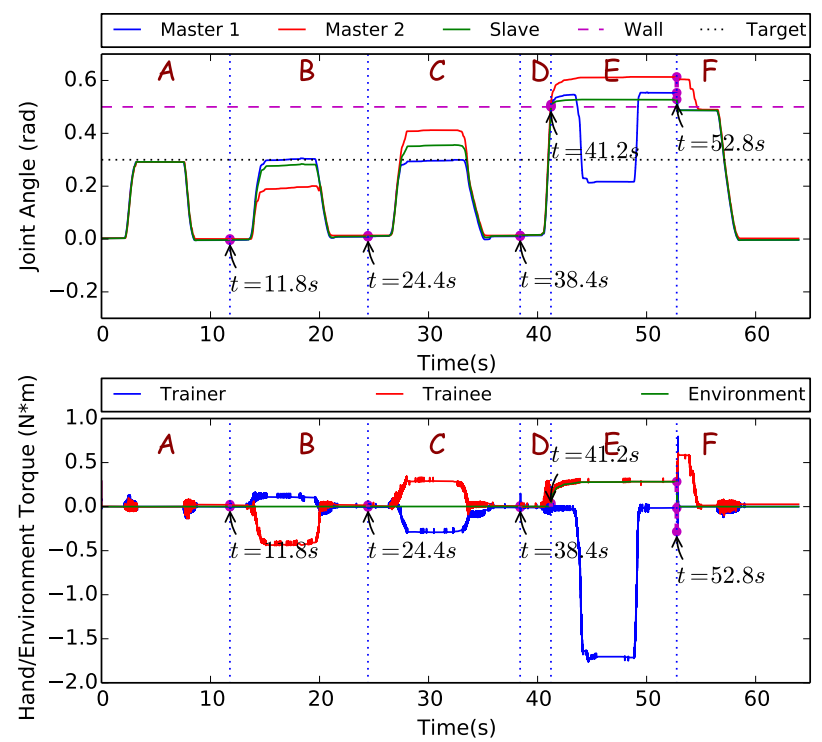

Fig. 4. Positions and forces tracking (in manual OAA mode)
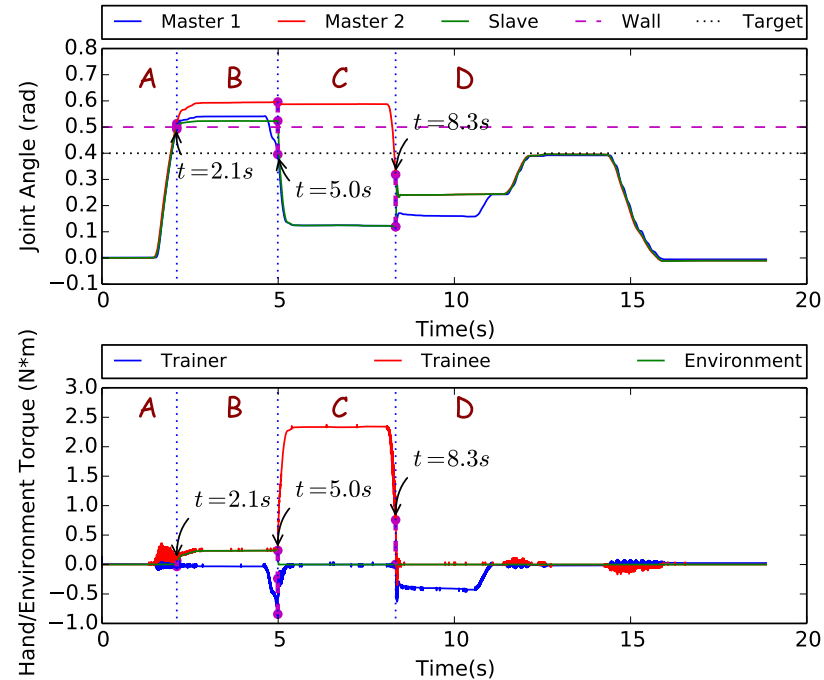

Fig. 5. Positions and forces tracking (in automatic OAA mode)

\section{B. Automatic $O A A$}

In this experiment, only the phases $\mathrm{D}, \mathrm{E}$ and $\mathrm{F}$ shown in previous one are considered. The difference is that automatic OAA takes effect. We set $B=0.04$ detailed in Eq. 3, which represents the allowed maximum position distance. The target position is $0.4 \mathrm{rad}$. The virtual wall keeps the same configuration as previously. Fig. 5 shows the positions and forces tracking. The slave establishes contact with the wall at time $2.1 \mathrm{~s}$. At time $4.8 \mathrm{~s}$, the trainer pulls back his device while the trainee maintains his own position.

As soon as the distance of positions crosses the boundary (at time $5 \mathrm{~s}$ ), the authority is automatically switched to the trainer ( $\alpha$ from 0 to 1 ). Thereafter the slave follows the trainer in period $\mathrm{C}$. The trainee experiences a resistant force since he tries to keep his position. The authority is automatically switched back to the trainee at time $8.3 \mathrm{~s}$, after he releases his position. The trainee guides the slave to the target position and finish the task in phase D. Notice that the automatic OAA could be enabled/disabled manually by the trainer.

\section{CONCLUSION}

In this paper, in order to provide a robust supervised hands-on training system, we designed a dual-user teleoperation system with Online Authority Adjustment (OAA). The stability of the system is ensured by an energetic modeling which guarantees the passivity of the system while the authority parameters evolve in time. The experiments revealed qualitatively a good transparency with both manual and automatic behaviors. Future work will extend the number of degrees of freedom and incorporate virtual fixture based guidance coordinated with OAA.

\section{REFERENCES}

[1] R. Taylor and D. Stoianovici, "Medical robotics in computer-integrated surgery," Robotics and Automation, IEEE Transactions on, vol. 19, pp. 765-781, Oct 2003.

[2] M. Tavakoli, R. Patel, and M. Moallem, Haptics For Teleoperated Surgical Robotic Systems. World Scientific, 2008.

[3] L. Panait, E. Akkary, R. Bell, K. Roberts, S. Dudrick, and A. Duffy, "The role of haptic feedback in laparoscopic simulation training," Journal of Surgical Research, vol. 156, pp. 312-316, Oct 2009.

[4] S. Nudehi, R. Mukherjee, and M. Ghodoussi, "A shared-control approach to haptic interface design for minimally invasive telesurgical training," Control Systems Technology, IEEE Transactions on, vol. 13, pp. 588-592, Jul 2005.

[5] B. Khademian and K. Hashtrudi-Zaad, "Shared control architectures for haptic training : performance and coupled stability analysis," The International Journal of Robotics Research, vol. 30, pp. 1627-1642, Mar 2011.

[6] A. Ghorbanian, S. Rezaei, A. Khoogar, M. Zareinejad, and K. Baghestan, "A novel control framework for nonlinear time-delayed dualmaster/single-slave teleoperation," ISA Transactions, vol. 52, pp. 268 277, Mar 2013.

[7] K. Razi and K. Hashtrudi-Zaad, "Analysis of coupled stability in multilateral dual-user teleoperation systems," Robotics, IEEE Transactions on, vol. 30, pp. 631-641, Jun 2014.

[8] M. Shahbazi, S. Atashzar, and R. Patel, "A dual-user teleoperated system with virtual fixtures for robotic surgical training," in Robotics and Automation (ICRA), 2013 IEEE International Conference on, pp. 3639-3644, May 2013.

[9] M. Shahbazi, H. Talebi, and R. Patel, "Networked dual-user teleoperation with time-varying authority adjustment : A wave variable approach," in Advanced Intelligent Mechatronics (AIM), 2014 IEEE/ASME International Conference on, pp. 415-420, Jul 2014.

[10] B. Maschke and A. van der Schaft, "Port controlled hamiltonian systems : modeling origins and system theoretic properties," in Nonlinear Control Systems (NOLCOS), Proceedings of the Third Conference on, 1992.

[11] S. Stramigioli, A. van der Schaft, B. Maschke, and C. Melchiorri, "Geometric scattering in robotic telemanipulation," Robotics and $\mathrm{Au}$ tomation, IEEE Transactions on, vol. 18, pp. 588-596, Aug 2002.

[12] C. Secchi, S. Stramigioli, and C. Fantuzzi, Control of Interactive Robotic Interfaces : A Port-Hamiltonian Approach. Springer, 2007.

[13] S. Stramigioli, Modeling and IPC Control of Interactive Mechanical Systems : A Coordinate-Free Approach. Springer, 2001.

[14] A. J. V. d. Schaft, L2-Gain and Passivity Techniques in Nonlinear Control. Springer, 1996.

[15] T. Sansanayuth, I. Nilkhamhang, and K. Tungpimolrat, "Teleoperation with inverse dynamics control for phantom omni haptic device," in SICE Annual Conference (SICE), 2012 Proceedings of, pp. 2121-2126, Aug 2012.

[16] S. Nicosia and P. Tomei, "Robot control by using only joint position measurements," Automatic Control, IEEE Transactions on, vol. 35, pp. 1058-1061, Sep 1990. 